\title{
Valoración del confort térmico de bovinos productores de carne en finalización intensiva en clima cálido
}

\author{
Evaluation of the thermal comfort of beef cattle in feedlot in hot weather
}

Citlaly Zazueta G. ${ }^{1}$, Isabel Castro P. ${ }^{1}$, Alfredo Estrada-Angulo ${ }^{1}$, Jesús Portillo L. ${ }^{1}$, David Urías E. ${ }^{1}$, Francisco Ríos R. ${ }^{1,2}$

\section{Resumen}

Se valoró el confort térmico de ganado bovino productor de carne en finalización intensiva, mediante la disponibilidad de sombra y espacio vital, en condiciones de clima semiseco muy cálido de la región costera del noroeste de México durante el otoño y el invierno y alojados en tres tipos de corral. El diseño de los corrales varió de acuerdo con la infraestructura disponible en la unidad de producción pecuaria (área de corral y sombra por animal). El periodo de observación fue de seis semanas por época. Se registró la temperatura ambiente y la humedad relativa diaria y se calculó el Índice de Temperatura y Humedad (ITH). Se midió el área de cada corral, el área de sombra y el número de bovinos por corral para calcular el espacio vital y la sombra disponible al inicio y al final del periodo de engorde. En otoño, el ganado se encontró en estado de Peligro a Emergencia térmica (ITH $\geq 84$ unidades) y en el invierno en estado de Alerta térmica (ITH $\geq 70$ ). La provisión de espacio vital y de sombra fue insuficiente en ambas épocas y periodos de engorda de acuerdo con indicadores para la especie bovina productora de carne en confinamiento, independientemente del diseño del corral.

Palabras clave: bovinos de carne, bienestar animal, ambiente térmico, instalaciones pecuarias

\footnotetext{
${ }^{1}$ Facultad de Medicina Veterinaria y Zootecnia, Universidad Autónoma de Sinaloa, México

${ }^{2}$ E-mail:fgrios@uas.edu.mx
}

Recibido: 15 de diciembre de 2020

Aceptado para publicación: 24 de agosto de 2021

Publicado: 27 de octubre de 2021

CLos autores. Este artículo es publicado por la Rev Inv Vet Perú de la Facultad de Medicina Veterinaria, Universidad Nacional Mayor de San Marcos. Este es un artículo de acceso abierto, distribuido bajo los términos de la licencia Creative Commons Atribución 4.0 Internacional (CC BY 4.0) [https:// creativecommons.org/licenses/by/4.0/deed.es] que permite el uso, distribución y reproducción en cualquier medio, siempre que la obra original sea debidamente citada de su fuente original 
The thermal comfort of beef cattle in intensive finishing was assessed through the availability of shade and living space, in conditions of the very hot semi-dry climate of the northwestern coastal region of Mexico during autumn and winter and housed in three types of pens. The design of the pens varied according to the infrastructure available in the livestock production unit (pen area and shade per animal). The observation period was six weeks per season. The daily ambient temperature and relative humidity were measured, and the Temperature and Humidity Index (ITH) was calculated. The area of each pen, the shade area, and the number of cattle per pen were measured to calculate the living space and the shade available at the beginning and at the end of the fattening period. In autumn, the cattle were in a state of Danger to Emergency Thermal (ITH $\geq 84$ units) and in the winter in a state of Thermal Alert (ITH $\geq 70$ ). The provision of living space and shade was insufficient in both seasons and periods according to indicators for confined meat-producing cattle, regardless of the pen design.

Key words: beef cattle, animal welfare, thermal environment, livestock facilities

\section{INTRODUCCIÓN}

La creciente demanda de proteína de origen animal intensifica el sistema de producción de carne bovina (Thornton, 2010), a costa de la disminución del espacio vital y del incremento de la densidad en los corrales de engorda, que limita la capacidad de los bovinos para expresar comportamientos naturales, a la vez que aumenta la incidencia de agresiones entre ellos (Lees et al., 2019; Romo-Valdez et al., 2019). De otra parte, la percepción de los consumidores acerca de las prácticas de producción y su impacto en la pérdida de bienestar de los animales influye en el desarrollo de esta actividad (Tucker et al., 2015).

De acuerdo con los principios del bienestar animal, el diseño de las instalaciones pecuarias debe minimizar el estrés y la tensión social, toda vez que la conducta y la organización social de los bovinos pueden ser afectadas por las condiciones de alojamiento y la disponibilidad de espacio vital (Huzzey et al., 2006; Gaughan et al., 2008). A la modificación del ambiente natural en los corrales de engorda, se suman condiciones climáticas adversas que inducen el estrés por calor que se asocia con la reducción de la productividad y pérdida de bienestar de los bovinos, principalmente durante los meses de verano (Lees et al., 2019), o cuando se intensifican las lluvias (Grandin, 2016). El estado de confort térmico de los bovinos se altera cuando la combinación de factores climáticos es desfavorable, por ello la recomendación del uso de sombras que ayuden a mitigar el efecto de la temperatura ambiental mayor de $25{ }^{\circ} \mathrm{C}$ $\mathrm{y}$ el efecto de la humedad relativa superior a 40\% (Mader et al., 2007; Arias et al., 2008). Se ha demostrado que proporcionar $2 \mathrm{~m}^{2} / \mathrm{ani}$ mal de sombra mejora el bienestar y el rendimiento del ganado bovino (Sullivan et al., 2011). Castro-Pérez et al. (2020) determinaron que aumentar el espacio de sombra en el corral de engorda incrementa los indicadores productivos; asimismo, Ha et al. (2018) afirman que el aumento en el espacio vital puede representar mejoras en el bienestar de los bovinos, ya que favorece que manifiesten su comportamiento natural, aumentan su conducta social y disminuyen las expresiones agonistas. 
Aunque los bovinos tienen la capacidad para adaptarse a las condiciones medioambientales, hay épocas del año en las que están expuestos al estrés por calor, o por las llamadas ondas de calor, que implican periodos de calor y humedad relativa inconfortable por periodos cortos o prolongados (BrownBrandl et al., 2006), ya sea debido a la oscilación de la temperatura ambiental, o bien cuando diversos factores se combinan generalmente durante un corto periodo de tiempo (Arias et al., 2008). El efecto de las condiciones ambientales en la respuesta productiva, parámetros fisiológicos y en los indicadores de bienestar durante el verano ha sido ampliamente reportado (Bernabucci et al., 2010; Gaughan et al., 2010; BrownBrandl et al., 2017; Ratnakaran et al., 2017). De igual manera, se ha demostrado el mayor impacto del estrés por calor durante el verano en el bienestar del ganado bovino (BrownBrandl y Jones, 2007; Gaughan et al., 2008; Mader et al., 2010; Lima et al., 2020); sin embargo, esta situación puede persistir a lo largo del año en regiones tropicales. Con base a esto, el objetivo del presente trabajo fue valorar el confort térmico de ganado bovino productor de carne en finalización intensiva mediante la disponibilidad de sombra y espacio vital en condiciones de clima semiseco muy cálido durante el otoño y el invierno.

\section{Materiales y Métodos}

\section{Ubicación y Tipo de Estudio}

El estudio se llevó a cabo en una Unidad de Producción Pecuaria (UPP) localizada en la zona costera central del estado de Sinaloa, México. El clima de la región $\left(\mathrm{BS}_{1}\left(\mathrm{~h}^{\prime}\right) \mathrm{w}(\mathrm{w}) \mathrm{e}\right.$, se define como semiseco muy cálido, extremoso con lluvias en verano, con temperatura media anual de $25.9^{\circ} \mathrm{C}$, mínima promedio de $10.5^{\circ} \mathrm{C}$ y máxima promedio de $36^{\circ} \mathrm{C}$, y humedad relativa promedio anual de 68\% (Estación Climatológica, EB-UAS, 2020).
El estudio fue de tipo observacional prospectivo (Manterola y Otzen, 2014), y se realizó durante seis semanas, a partir de la tercera a la octava semana del otoño y durante seis semanas a partir de la tercera a la octava semana del invierno. En cada época se realizaron visitas diarias a seis corrales de finalización intensiva elegidos por conveniencia con base a una programación aleatorizada. Las observaciones fueron registradas por una sola persona.

\section{Variables Climáticas}

La temperatura ambiental y la humedad relativa fueron registrados mediante termohigrómetros digitales (Avaly Taylor VAEDT-1-55, CDMX) colocados dentro de cada corral objeto de estudio. El índice de temperatura y humedad fue calculado usando la fórmula: $\mathrm{ITH}=(0.8 \times \mathrm{T})+[(\mathrm{HR} / 100) \mathrm{x}$ (T-1.4) ] + 46.4 (Mader et al., 2006), donde T es la temperatura ambiental en grados Celsius y HR es la humedad relativa expresada en porcentaje.

\section{Instalaciones}

En la UPP se engorda anualmente un promedio de 47967 cabezas de ganado bovino en 2.2 ciclos. La infraestructura consta de 202 corrales para producción distribuidos en ocho secciones, 29 corrales de reciba y tres de enfermería. Los bovinos se alojaron en corrales convencionales para la engorda y finalización, construidos con similitud de materiales: tubería metálica de $1.60 \mathrm{~m}$ de altura, piso de tierra, sombra provista a base de material metálico colocada a $3.0 \mathrm{~m}$ de altura, bebedero automático de acero inoxidable compartido por dos corrales y banqueta de $2 \mathrm{~m}$ de ancho en el área de comedero lineal. Las características particulares de los corrales se muestran en el Cuadro 1. Para propósitos de la investigación, los corrales se identificaron mediante la siguiente nomenclatura: Diseño 1 (DC1), Diseño 2 (DC2) y Diseño 3 (DC3), que corresponden a las secciones 8,1 y 3 , respectivamente. De cada 
Cuadro 1. Características de los corrales de finalización intensiva en la Unidad de Producción Pecuaria conforme al diseño específico (Sinaloa, México)

\begin{tabular}{|c|c|c|c|}
\hline Características & $\begin{array}{l}\text { Diseño de corral } 1 \\
\text { (DC1) }\end{array}$ & $\begin{array}{l}\text { Diseño de corral } 2 \\
\text { (DC2) }\end{array}$ & $\begin{array}{c}\text { Diseño de corral } 3 \\
\text { (DC3) }\end{array}$ \\
\hline Superficie disponible, $\mathrm{m}^{2}$ & 1620 & 1200 & 1080 \\
\hline Área de sombra, $\mathrm{m}^{2}$ & 360 & 171 & 180 \\
\hline Altura de la sombra, m & 3.0 & 3.0 & 3.0 \\
\hline Tipo de sombra & Metálica & Metálica & Metálica \\
\hline Orientación de la sombra & $\mathrm{E}$ a O & $\mathrm{Na} \mathrm{S}$ & $\mathrm{E}$ a O \\
\hline Comedero disponible, $\mathrm{m}$ & 56 & 30 & 36 \\
\hline Bebedero disponible, $\mathrm{m}$ & 6.10 & 6.10 & 6.10 \\
\hline
\end{tabular}

una de estas secciones, se seleccionaron los mismos dos corrales para cada época objeto de estudio.

\section{Animales}

Los bovinos del estudio presentaban las características típicas de los corrales de engorda tecnificada en el norte de México: machos sin castrar, con un componente genético de aproximadamente $60 \%$ Bos indicus en cruzamiento con Bos taurus, principalmente de las razas Pardo Suizo Americano, Pardo Suizo Europeo, Beefmaster, Charolais y Angus, en proporciones no determinadas. Dependiendo de la capacidad del corral y una vez que se han realizado todas las prácticas profilácticas, este se cierra de dos a tres días posteriores al arribo del ganado.

El protocolo de manejo y alimentación de los bovinos fue el que comúnmente se sigue en las engordas tecnificadas de la zona. El manejo consiste en vacunación, desparasitación y colocación de implantes (acetato de trembolona, estradiol y tilosina). El alimento se proporciona dos veces al día de acuerdo con un programa de seis dietas que básicamente incluyen maíz hojuelado, paja de maíz, pasta de soya, granos secos de destilería, melaza y premezcla mineral. Las mediciones se realizaron en los corrales que se encontraban en la etapa de finalización y que coincide con el inicio del suministro de la dieta cuatro de las seis que integran el programa de alimentación.

\section{Procedimiento}

En los corrales de engorda se midió la superficie del corral, orientación y altura de la sombra, longitud de bebedero y comedero, y se registró el tipo de diseño. Al inicio y al final del periodo de engorda se registró el número y peso de los bovinos por corral, así como el espacio vital. Para esto último, se dividió el área de la superficie del corral $\left(\mathrm{m}^{2}\right)$ entre el número de bovinos alojados en el corral. El espacio de sombra disponible se calculó dividiendo la superficie total de sombra $\left(\mathrm{m}^{2}\right)$ entre el número de bovinos en el corral. Estos resultados fueron contrastados con las especificaciones de espacio vital y de sombra disponible para bovinos productores de carne en finalización intensiva propuestos por Lagos et al. (2014). 


\section{Análisis Estadístico}

El corral de engorda fue la unidad de observación. Para los valores de temperatura, humedad relativa e ITH se calculó la media, valores mínimos y máximos por semana de observación y el periodo general. Los valores de ITH fueron transformados a rangos con el procedimiento RANK (SAS, 2002), debido a que las varianzas de los datos de la combinación periodo, diseño y hora fueron heterogéneas. Se realizó análisis de la varianza de los rangos de ITH con el modelo lineal general que incluyó los efectos de periodo del año (otoño, invierno), diseño de corral (DC1, DC2, DC3), hora de medición $(8,12$, $16 \mathrm{~h}$ ), así como, las interacciones de primer y segundo orden. Se obtuvieron las medias de cuadrados mínimos (opción LSMEANS en Proc GLM de SAS) y la comparación de puntuaciones de rangos se realizó con la prueba de Dunn (Bonferroni) (SAS, 2002). El valor de alfa máximo para considerar diferencia estadística fue de 0.05 . Los resultados se muestran como promedios y error estándar y gráficas de interacción.

\section{Resultados y Discusión}

\section{Condiciones Climáticas Generales}

Las condiciones climáticas de los corrales de engorda se referenciaron con la información provista por el Servicio Meteorológico Nacional de la Comisión Nacional del Agua (CONAGUA, s.f.), cuyos resultados mostraron que en otoño la temperatura máxima fue de $32.8{ }^{\circ} \mathrm{C}$, la mínima de $18.2{ }^{\circ} \mathrm{C}$, humedad relativa de $71 \%$ e ITH máximo de 86 unidades, mientras que en invierno la temperatura máxima fue de $28.3{ }^{\circ} \mathrm{C}$, mínima de $22.2{ }^{\circ} \mathrm{C}$, humedad relativa $71 \%$ e ITH máximo de 79 unidades.

\section{Estación de Otoño}

En el Cuadro 2 se presentan los promedios de la temperatura ambiental, humedad relativa e índice de temperatura y humedad en los corrales de finalización intensiva durante el otoño. El promedio general del ITH fue de $80.5 \mathrm{y}$ el promedio máximo de 87.9, por lo que se considera el ganado bovino se encontró bajo estas condiciones en un estado de Peligro a Emergencia térmica (ITH $\geq 84$ unidades), conforme a las categorías de estrés térmico establecido por Nienaber y Hahn (2007). Al respecto, el ITH se considera como un indicador de la carga térmica en el ganado bovino productor de carne en confinamiento intensivo (Gaughan et al., 2008). Estos resultados muestran que se está comprometiendo el estado de confort térmico del ganado bovino. Según Renaudeau et al. (2012), los animales sufren inconvenientes debido a la pérdida del balance térmico, pudiéndose afectar la función productiva. En este sentido, Gaughan et al. (2003) sugieren el uso del índice de carga de calor acumulado (HLIhoras) para proporcionar una mejor guía del estado térmico del ganado en las condiciones ambientales del lugar en un momento específico del día.

Se ha establecido que los bovinos tienen un mejor desempeño en una zona termo neutral de $20^{\circ} \mathrm{C}\left(10-26{ }^{\circ} \mathrm{C}\right)$; sin embargo, cuando la temperatura ambiental excede los $27{ }^{\circ} \mathrm{C}$ y, especialmente, si la humedad relativa es mayor a $40 \%$, se rompe el equilibrio de la zona termo neutral debido a la condición homeoterma de los bovinos, por lo que en tal situación se pierde el bienestar y se reduce el potencial productivo. Bajo estas condiciones climáticas, el bovino aumenta la sudoración y la tasa de respiración hasta llegar al jadeo, mediante las cuales libera calor a través de la evaporación (Mader et al., 2006; Arias et al., 2008). A diferencia de la temperatura, 
Cuadro 2. Promedio de la temperatura ambiental, humedad relativa e índice de temperatura y humedad en los corrales de finalización intensiva en el otoño (Sinaloa, México)

\begin{tabular}{cccccccccc}
\hline \multirow{2}{*}{ Semana } & \multicolumn{3}{c}{ Temperatura $\left({ }^{\circ} \mathrm{C}\right)$} & \multicolumn{3}{c}{ Humedad relativa $(\%)$} & \multicolumn{3}{c}{ ITH } \\
\cline { 2 - 9 } & Mín. & Máx. & Media & Mín. & Máx. & Media & Mín. & Máx. & Media \\
\hline 1 & 27.3 & 43.1 & 35.0 & 17 & 70 & 34.9 & 72.3 & 88.1 & 81.6 \\
2 & 26.1 & 40.8 & 33.4 & 21 & 72 & 34.7 & 70.6 & 88.9 & 80.1 \\
3 & 26.1 & 43.7 & 35.5 & 20 & 64 & 30.1 & 70.6 & 88.2 & 81.3 \\
4 & 28.0 & 45.0 & 36.1 & 20 & 49 & 25.4 & 71.8 & 94.8 & 81.0 \\
5 & 29.8 & 39.7 & 34.9 & 19 & 41 & 24.4 & 74.1 & 83.6 & 79.6 \\
6 & 29.0 & 38.2 & 33.5 & 18 & 65 & 30.9 & 73.4 & 83.9 & 79.3 \\
\hline General & 27.7 & 41.7 & 34.7 & 19 & 60 & 30.0 & 72.1 & 87.9 & 80.5 \\
\hline
\end{tabular}

ITH: Índice de temperatura y humedad. Mín.: Mínimo, Máx.: Máximo

que generalmente varía considerablemente entre la noche y el día, el punto de rocío tiende a cambiar lentamente, así es que aunque la temperatura baje por la noche, en un día húmedo, generalmente la noche es húmeda. En este sentido, el valor de la humedad relativa máxima, particularmente en esta región costera del noroeste de México disminuye rápidamente, de $95 \%$ a fines del verano a $58 \%$ a principios de otoño, hasta llegar a $14 \%$ en noviembre. Considerando lo anterior, el periodo más húmedo del año en esta región dura 6.1 meses (14 de mayo al 17 de noviembre), y durante ese tiempo el nivel de confort se considera bochornoso, opresivo o insoportable por lo menos durante el $27 \%$ del tiempo (Estación climatológica, Escuela de Biología UAS, 2020).

Los resultados de disponibilidad de espacio vital y sombra en los corrales de finalización de ganado bovino al inicio y al final de la engorda se muestran en el Cuadro 3. El espacio vital en la etapa inicial de la engorda en los corrales DC1 fue superior en $8 \%$ al recomendado, pero inferior en 29 y $28 \%$ para corrales DC2 y DC3, en tanto que en la etapa final de la engorda fue de 25, 34 y $41 \%$ inferior al espacio vital recomendado en los corrales DC1, DC2 y DC3, respectivamente. El espacio vital recomendado para bovinos menores de $300 \mathrm{~kg}$ es de $15 \mathrm{~m}^{2}$ y para bovinos de $400 \mathrm{~kg}$ en adelante es de $20 \mathrm{~m}^{2}$ (Lagos et al., 2014).

Estos resultados demuestran que el manejo del espacio vital en la UPP no está predefinido; es decir, no se tiene en consideración la capacidad del corral de engorda en función de las necesidades de espacio vital de los bovinos ni en la etapa inicial ni en la etapa de finalización, independientemente de la estación del año. A este respecto, existe una tendencia mundial de reducir el espacio vital en corrales de bovinos de finalización intensiva con la finalidad de incrementar la rentabilidad de la unidad de producción; sin embargo, la reducción del espacio vital afecta el comportamiento animal y afecta al bienestar de los bovinos (Macitelli et al., 2020).

El mínimo de sombra recomendado para bovinos productores de carne en confinamiento es de $3.7 \mathrm{~m}^{2}$ (Lagos et al., 2014), siendo que los resultados de disponibilidad de sombra en la etapa inicial de la engorda fue de 
Cuadro 3. Disponibilidad de espacio vital y sombra de acuerdo con el diseño de corral ${ }^{1}$ de finalización de ganado bovino, al inicio y final de la engorda en la época de otoño (Sinaloa, México)

\begin{tabular}{lcccccc}
\hline \multirow{2}{*}{ Característica } & \multicolumn{3}{c}{ Etapa inicial } & \multicolumn{3}{c}{ Etapa final } \\
\cline { 2 - 7 } & DC1 & DC2 & DC3 & DC1 & DC2 & DC3 \\
\hline $\begin{array}{l}\text { Número de bovinos } \\
\text { Peso vivo, kg }\end{array}$ & 100 & 112 & 100 & 108 & 91 & 91 \\
$\begin{array}{l}\text { Espacio vital/bovino, } \mathrm{m}^{2} \\
\text { Diferencia espacio vital, }\end{array}$ & 16.2 & 10.71 & 10.80 & 15 & 13.18 & 11.86 \\
$\begin{array}{l}\text { m } \\
\text { Sombra }\end{array}$ & 1.2 & -4.29 & -4.20 & -5.0 & -6.81 & -8.13 \\
$\begin{array}{l}\text { disponible/bovino, } \mathrm{m}^{2} \\
\begin{array}{l}\text { Diferencia sombra } \\
\text { disponible }\end{array}\end{array}$ & 3.6 & 1.52 & 1.8 & 3.30 & 1.87 & 1.97 \\
\hline
\end{tabular}

DC1: diseño de corral 1; DC2: diseño de corral 2; DC3: diseño de corral 3

1 Dos corrales por cada tipo de corral y en cada estación

2 Diferencia de sombra disponible con respecto a $3.7 \mathrm{~m}^{2} /$ cabeza (Lagos et al., 2014)

$2.7,53.2$ y $45.9 \%$ inferior a dichas recomendaciones en los corrales DC1, DC2 y DC3, respectivamente. Sullivan et al. (2011) observaron mejora en los indicadores de bienestar en el ganado bovino al proporcionar sombra a razón de 3.3 vs $2.0 \mathrm{~m}^{2} /$ cabeza.

En la etapa final de la engorda, la sombra disponible es $65 \%$ mayor en los corrales DC1, 6.5\% menor en los corrales DC2 y $24 \%$ menor en los corrales DC3. Al respecto, Hahn (1985) refiere que proporcionar entre 1.8 a $2.5 \mathrm{~m}^{2}$ de sombra por cabeza puede ser causa de hacinamiento y no representa un valor adecuado para corrales de engorda en ambientes tropicales. $\mathrm{Al}$ respecto, si las condiciones en el corral de engorda son desfavorables; es decir, el espacio vital es menor para expresar su comportamiento social natural y de manera intuitiva otros comportamientos deseables, los bovinos pueden desarrollar comportamientos agresivos y anormales
(Pelley et al., 1995); y si a esto se le suma la deficiencia en la disponibilidad de sombra en condiciones ambientales adversas, los bovinos disminuyen el tiempo dedicado a consumir alimento y el que permanecen echados, aumenta el tiempo dedicado a beber agua y el que permanecen de pie cerca de los bebederos; también se pueden observar cambios en la distribución del ganado dentro de los corrales, pues tienden a permanecer más tiempo en lugares con mejor ventilación (Arias et al., 2008).

En los casos donde el área de sombra no es suficiente para albergar a todos los bovinos en un corral, varios de ellos permanecerán desprotegidos y sometidos a estrés por calor. En estos casos es conveniente observar el comportamiento de los animales y registrar la distancia media entre ellos, lo cual puede ser de utilidad para la planificación de los corrales (Gomes da Silva, 2010). 
Cuadro 4. Promedio de la temperatura ambiental, humedad relativa e índice de temperatura y humedad en los corrales de finalización intensiva en el invierno (Sinaloa, México)

\begin{tabular}{cccccccccc}
\hline & \multicolumn{3}{c}{ Temperatura $\left({ }^{\circ} \mathrm{C}\right)$} & \multicolumn{3}{c}{ Humedad relativa $(\%)$} & \multicolumn{3}{c}{ ITH } \\
\hline Semana & Mín. & Máx. & Media & Mín. & Máx. & Media & Mín. & Máx. & Media \\
\hline 1 & 11.8 & 32.1 & 26.4 & 25.5 & 72.2 & 31.8 & 60.4 & 78.2 & 71.3 \\
2 & 7.9 & 32.9 & 27.3 & 23.5 & 75.0 & 28.7 & 55.3 & 80.6 & 71.9 \\
3 & 7.0 & 37.7 & 28.6 & 21.5 & 80.4 & 30.4 & 67.4 & 81.0 & 73.7 \\
4 & 7.1 & 38.5 & 26.3 & 21.0 & 83.7 & 39.0 & 66.1 & 78.8 & 72.2 \\
5 & 7.1 & 38.0 & 30.3 & 21.0 & 83.4 & 26.5 & 69.2 & 79.1 & 75.1 \\
6 & 7.1 & 38.5 & 25.8 & 21.0 & 83.7 & 35.8 & 67.5 & 75.1 & 71.2 \\
\hline General & 8.1 & 36.2 & 27.4 & 22.3 & 79.5 & 32.0 & 64.3 & 78.8 & 72.5 \\
\hline
\end{tabular}

ITH: Índice de temperatura y humedad. Mín.: Mínimo, Máx.: Máximo

\section{Estación de Invierno}

Los promedios de la temperatura ambiental, humedad relativa e índice de calor y humedad durante el invierno se muestran en el Cuadro 4. El valor promedio general del ITH excedió ligeramente las 72 unidades (72.5), por lo que de acuerdo con lo propuesto por Nienaber y Hahn (2007) el ganado bovino se encuentra en un estado de Alerta térmica (ITH $\geq 70)$.

Aunque existen otros factores que contribuyen a potenciar o disminuir el estrés calórico en el ganado bovino, los de mayor importancia son la velocidad del viento, la radiación solar, la temperatura ambiental y la humedad relativa (Arias et al., 2008; Olivares et al., 2013). No obstante, Gaughan et al. (2008) refieren que el ITH se considera como el indicador de la carga térmica más relevante para el ganado bovino.

De acuerdo con lo observado en el Cuadro 5 para la época de invierno, el espacio vital en la etapa inicial de la engorda fue de 10,19 y $25 \%$ inferior al recomendado en los corrales DC1, DC2 y DC3, y en la etapa fi- nal de la engorda de 25, 33 y $38 \%$ inferior en corrales DC1, DC2 y DC3, respectivamente. Estos cálculos se basan en el requerimiento de espacio vital recomendado por Lagos et al. (2014). La reducción del espacio vital en el corral de finalización trae consigo la disminución de las actividades habituales del ganado (Ha et al., 2108) a medida que se incrementa el peso de los bovinos durante el transcurso de la engorda. En cambio, beneficios económicos y menor morbilidad se han registrado cuando se incrementa la disponibilidad de espacio vital (Montelli et al., 2019); que en suma contribuye a la mejora de los indicadores de bienestar del ganado bovino en el corral de engorda.

Respecto a la sombra disponible por bovino, en la etapa inicial de la engorda el área disponible fue de 19,54 y $51 \%$ inferior a las recomendaciones en los corrales DC1, DC2 y DC3, respectivamente, en tanto que en la etapa final fue de $8.1,48$ y $43 \%$ en los corrales DC1, DC2 y DC3, respectivamente, considerando que el mínimo de sombra recomendado para bovinos productores de carne en confinamiento es de $3.7 \mathrm{~m}^{2}$ (Lagos et al., 2014). Blaine y Nsahlai (2011), en épo- 
Cuadro 5. Disponibilidad de espacio vital y sombra de acuerdo con el diseño de corral de finalización de ganado bovino, al inicio y final de la engorda en la época de invierno (Sinaloa, México)

\begin{tabular}{lcccccc}
\hline \multirow{2}{*}{ Característica } & \multicolumn{3}{c}{ Etapa inicial } & \multicolumn{3}{c}{ Etapa final } \\
\cline { 2 - 7 } & DC1 & DC2 & DC3 & DC1 & DC2 & DC3 \\
\hline Número de bovinos & 120 & 99 & 96 & 108 & 90 & 87 \\
Peso vivo, kg & $233.5 \pm 1$ & $256 \pm 11.3$ & $285.5 \pm 10.6$ & $491 \pm 18.4$ & $488 \pm 2.8$ & $500.5 \pm 23.3$ \\
Espacio vital/bovino, $\mathrm{m}^{2}$ & 13.5 & 12.1 & 11.2 & 14.9 & 13.3 & 12.4 \\
$\begin{array}{l}\text { Diferencia espacio vital, } \\
\mathrm{m}^{2}\end{array}$ & -1.5 & -2.9 & -3.8 & -5.1 & -6.7 & -7.6 \\
$\begin{array}{l}\text { Sombra } \\
\text { disponible/bovino, } \mathrm{m}^{2}\end{array}$ & 3.0 & 1.7 & 1.8 & 3.3 & 1.9 & 2.06 \\
$\begin{array}{l}\text { Diferencia sombra } \\
\text { disponible }{ }^{1}, \mathrm{~m}^{2}\end{array}$ & -0.7 & -2.0 & -1.9 & -0.3 & -1.8 & -1.6 \\
\hline
\end{tabular}

DC1: diseño de corral 1; DC2: diseño de corral 2; DC3: diseño de corral 3

${ }^{1}$ Diferencia de sombra disponible con respecto a $3.7 \mathrm{~m}^{2} /$ cabeza (Lagos et al., 2014)

ca de invierno, proporcionaron $2.87 \mathrm{~m}^{2}$ de sombra por cabeza, proveniente de lámina de fierro corrugada, colocada a $5 \mathrm{~m}$ de altura y observaron mejora en los indicadores de bienestar de los bovinos. El estrés por calor es una preocupación importante para el bienestar animal porque reduce el rendimiento en el ganado bovino alojado en el corral de engorda durante la fase final del período de alimentación (Hagenmaier et al., 2016).

El análisis de la varianza (Cuadro 6) indica variación del ITH por efecto de la estación del año y hora del día ( $\mathrm{p}<0.01)$, así como por la interacción diseño de corral $\mathrm{x}$ hora $\mathrm{y}$ estación $\mathrm{x}$ diseño de corral $(\mathrm{p}<0.01)$; sin embargo, no muestra efecto del diseño del co$\operatorname{rral}(\mathrm{p}>0.05)$.

El valor de ITH a las 08:00 es inferior $(\mathrm{p}<0.01)$ al registrado a las $12: 00$ y a las $16: 00$ h $(72.8 \pm 0.31$ vs $78.4 \pm 0.30)$. En otoño el ITH fue superior en 7.8 unidades ( $80.4 \pm 0.21$ vs $72.6 \pm 0.19)$, con respecto al invierno $(\mathrm{p}<0.01)$. Los resultados confirman que los bovinos se encontraban en riesgo térmico, estando en otoño en la categoría de peligro térmico (ITH $>79 \leq 84)$ y en invierno en la categoría de alerta térmica (ITH $>70 \leq 79$ ). La interacción diseño de corral $\mathrm{x}$ hora de observación (Figura 1) se debió mayormente al diferente promedio de ITH a las 08:00. Es posible que la ubicación de los corrales favorezca ciertas condiciones de microclima. $\mathrm{La}$ interacción de estación del año x diseño de corral se debe a la intercepción que hay en las líneas de ITH para el DC2 y DC3 (Figura 2), efecto que se atribuye a la época del año, considerándose esta interacción un efecto fortuito.

Edwards-Callaway et al. (2021) afirman que el ganado bovino es susceptible a estrés por calor si no lo pueden disipar cuando se presentan temperaturas ambientales elevadas; asimismo, hacen referencia a diversos factores que impactan la susceptibilidad al estrés, muchos de los cuales se rela- 
Cuadro 6. Valor de ITH en corrales de bovinos en finalización intensiva según la estación del año, diseño del corral y hora del día (Sinaloa, México)

\begin{tabular}{|c|c|c|c|c|c|c|}
\hline \multirow{2}{*}{ Hora } & \multirow{2}{*}{ Estación } & \multicolumn{3}{|c|}{ Diseño de corral } & \multirow{2}{*}{$\begin{array}{c}\text { Hora- } \\
\text { Estación }\end{array}$} & \multirow[t]{2}{*}{ Hora } \\
\hline & & DC1 & $\mathrm{DC} 2$ & $\mathrm{DC} 3$ & & \\
\hline \multirow[t]{2}{*}{ 08:00 } & Otoño & 78.4 & 74.3 & 76.7 & 76.5 & $72.8 \pm 0.31^{b}$ \\
\hline & Invierno & 70.3 & 68.0 & 68.8 & 69.0 & \\
\hline \multirow[t]{2}{*}{$12: 00$} & Otoño & 82.3 & 83.3 & 82.6 & 82.7 & $78.8 \pm 0.29^{\mathrm{a}}$ \\
\hline & Invierno & 74.3 & 75.2 & 74.8 & 74.8 & \\
\hline \multirow[t]{2}{*}{$16: 00$} & Otoño & 81.7 & 82.7 & 81.7 & 82.0 & $78.0 \pm 0.31^{\mathrm{a}}$ \\
\hline & Invierno & 73.5 & 74.7 & 73.9 & 74.0 & \\
\hline \multirow[t]{2}{*}{ Estación } & Otoño & 80.8 & 80.1 & 80.3 & $80.4 \pm 0.21^{\mathrm{A}}$ & \\
\hline & Invierno & 72.7 & 72.6 & 72.5 & $72.6 \pm 0.19^{\mathrm{B}}$ & \\
\hline \multicolumn{2}{|c|}{ Diseño de corral } & $76.8 \pm 0.31^{\mathrm{a}}$ & $76.4 \pm 0.38^{\mathrm{a}}$ & $76.4 \pm 0.34^{\mathrm{a}}$ & & \\
\hline \multicolumn{5}{|c|}{ Probabilidad } & & \\
\hline \multicolumn{4}{|c|}{ Estación del año } & 0.01 & & \\
\hline \multicolumn{4}{|c|}{ Diseño de corral } & 0.22 & & \\
\hline \multicolumn{4}{|c|}{ Hora del día } & 0.01 & & \\
\hline \multicolumn{4}{|c|}{ Estación del año x Diseño de corral } & 0.01 & & \\
\hline \multicolumn{4}{|c|}{ Estación del año x Hora del día } & 0.26 & & \\
\hline \multicolumn{4}{|c|}{ Diseño de corral x Hora del día } & 0.01 & & \\
\hline \multicolumn{4}{|c|}{ Estación del año x Diseño de corral x Hora del día } & 0.06 & & \\
\hline
\end{tabular}

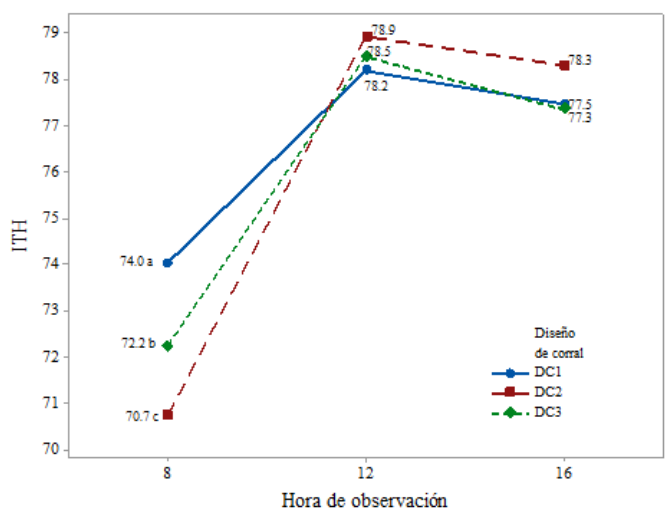

Figura 1. Efecto de la interacción Diseño del corral x Hora de observación en el valor del ITH en el corral de finalización de ganado bovino $(\mathrm{p}<0.01)$ cionan con el sistema de producción; es decir, disponibilidad de la sombra, microclima del medio ambiente y la nutrición del ganado.

\section{Conclusiones}

- El confort térmico durante el otoño e invierno de los bovinos productores de carne bajo las condiciones ambientales en Sinaloa, México, determinado se encuentra comprometido.

- Las limitaciones en espacio vital y disponibilidad de sombra no favorecen un ambiente térmico apropiado para el bienestar de los bovinos en el corral de engorda. 


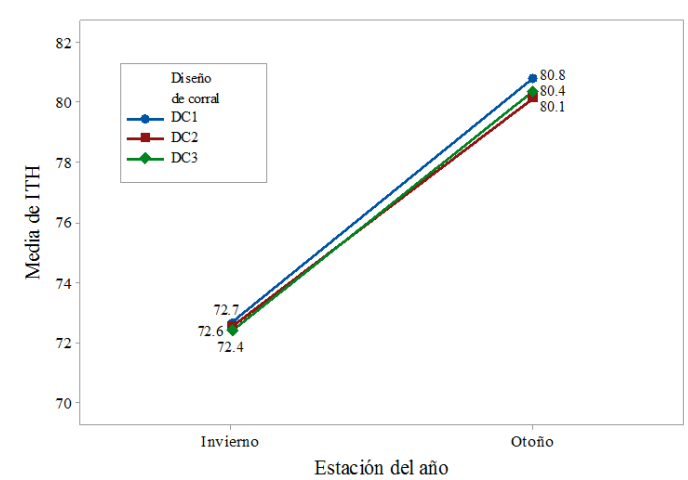

Figura 2. Efecto de la interacción Diseño del corral y Estación del año en el valor de ITH en el corral de finalización de ganado bovino $(\mathrm{p}<0.01)$

\section{Literatura Citada}

1. Arias RA, Mader TL, Escobar PC. 2008. Factores climáticos que afectan el desempeño productivo del ganado bovino de carne y leche. Arch Med Vet 40: 7-22. doi: 10.4067/S0301-732X2008000100002.

2. Bernabucci V, Lacetera N, Baumgard LH, Rhoads RP, Ronchi B, Nardone A. 2010. Metabolic and hormonal acclimation to heat stress in domesticated ruminants. Animal 4: 1167-1183. doi: 10.1017/S175173111000090X

3. Blaine KL, Nsahlai IV. 2011. The effects of shade on performance, carcass classes and behaviour of heatstressed feedlot cattle at the finisher phase. Trop Anim Health Prod 43: 609615. doi: 10.1007/s11250-010-9740-x

4. Brown-Brandl TM, Nienaber JA, Eigenberg RA, Mader TL, Morrow JL, Dailey JW. 2006. Comparison of heat tolerance of feedlot heifers of different breeds. Livest Sci 105: 19-26. doi: 10.1016/j.livsci.2006.04.012

5. Brown-Brandl TM, Jones DD. 2007. Development and validation of an animal susceptibility Model. ASABE Annual International Meeting. Paper $\mathrm{N}^{\circ} 074081$. Minneapolis, Minnesota.
6. Brown-Brandl TM, Chitko-McKown CG, Eigenberg RA, Mayer JJ, Welsh Jr TH, Davis JD, Purswell JL. 2017. Physiological responses of feedlot heifers provided access to different level of shade. Animal 11: 1344-1353. doi: 10.1017/S1751731116002664

7. Castro-Pérez BI, Estrada-Angulo A, Ríos-Rincón FG, Núñez-Benítez VH, Rivera-Méndez CR, Urías-Estrada JD, Zinn RA, et al. 2020. The influence of shade allocation or total shade plus overhead fan on growth performance, efficiency of dietary energy utilization, and carcass characteristics of feedlot cattle under tropical ambient conditions. Asian Austral J Anim 33: 1034-1041 . doi: 10.5713/ajas.19.0112

8. [CONAGUA] Comisión Nacional del Agua. s.f. Servicio Meteorológico Nacional. Normales climatológicas del Estado de Sinaloa. Periodo 1951-2010. [Internet]. Disponible en: https:// smn.conagua.gob.mx/es/informacionclimatologica-por-estado?estado $=$ sin

9. Edwards-Callaway LN, Cramer MC, Cadaret CM, Bigler EJ, Engle TE, Wagner JJ, Clark DL. 2021. Impact of shade on cattle well-being in the beef supply chain. J Anim Sci 1: 99:skaa375. doi: 10:1093/jas/skaa/375

10. Estación Climatológica de la Escuela de Biología. Universidad Autónoma de Sinaloa. 2020. [Internet] [13 marzo 2020]. Disponible en: https:// www.uas.edu.mx/servicios/clima/boletines

11. Gaughan J, Goopya J, Spark J. 2003. Excessive heat load index for feedlot cattle. Meat and Livestock Australia Ltd. North Sydney NSW.

12. Gaughan JB, Mader TL, Holt SM, Lisle A. 2008. A new heat index for feedlot cattle. J Anim Sci 86: 226-234. doi: $10.2527 /$ jas. 20070305

13. Gaughan JB, Bonner S, Loxton I, Mader TL, Lisle A, Lawrence R. 2010. Effect of shade on body temperature and performance of feedlot steers. J Anim Sci 88: 4056-4067. doi: 10.2527/jas2010-2987 
14. Gomes da Silva R. 2010. Wheater and climate and animal production. In: Guide to agricultural meteorological practices. World Metereological Organization. No. 134. Geneva, Switzerland.

15. Grandin T. 2016. Evaluation of the welfare of cattle housed in outdoor feedlot pens. Vet Anim Sci 1: 23-28. doi: 10.1016/j.vas2016.11.001

16. Ha JJ, Yang KL, Oh DY, Yi JK, Kim JJ. 2018. Rearing characteristics of fattening Hanwoo steers managed in different stocking densities. Asian Austral J Anim 31: 1714-1720. doi: 10.5713/ ajas.17.0451

17. Hagenmaier JA, Reinhardt CD, Bartle SJ, Thomson DU. 2016. Effect of shade on animal welfare, growth performance and carcass characteristics in large pens of beef cattle fed a beta agonist in a commercial feedlot. J Anim Sci 94: 5064-8076. doi: 10.2527/jas20160935

18. Hahn GL. 1985. Management and housing of farm animals in hot environments. In: Stress physiology of livestock. Vol. 2, Yousef MK (ed). Boca Raton, USA: CRC Press. p 151-174.

19. Huzzey JM, DeVries TJ, Valois P, Von Keyserlingk MA. 2006. Stocking density and feed barrier design affect the feeding and social behavior of dairy cattle. J Dairy Sci 81: 126-133. doi: 10.3168/jds.S0022-0302(06)72075-6

20. Lagos GH, González FJ, Castillo FR. 2014. Paquete tecnológico para la engorda de ganado bovino en corral. México: INIFAP. $61 \mathrm{p}$.

21. Lees AM, Sejian, V, Wallage AL, Steel CC, Mader TL, Lees JC, Gaughan JB. 2019. The impact of heat load on cattle: review. Animals 9:3 22-341. doi: 10.3390/ani9060322

22. Lima S, Bonvino SN, Pires BV., Bonilha SFM, Cyrillo NSG, Negrão JA, Paz CCP. 2020. Effect of high temperature on physiological parameters of Nelore (Bos taurus indicus) and Caracu (Bos taurus taurus) cattle breeds. Trop Anim Health Prod 52: 2233-
2241. doi.org/10.1007/s11250-02002249-y

23. Macitelli F, Braga JS, Gellatly D, Paranhos da Costa MJR. 2020. Reduced space in outdoor feedlot impacts beef cattle welfare. Animal 14: 2588-2597. doi: 10.1017/S1751731120001652

24. Mader TL, Davis MS, Brown-Brandl T. 2006. Environmental factors influencing heat stress in feedlot cattle. J Anim Sci 84: 712-719. doi: 10.2527/ 2006.843712x

25. Mader T, Griffin D, Hahn L. 2007. Managing feedlot heat stress. Institute of Agriculture and Natural Resourcer. University of Nebraska-Lincoln. [Internet]. Available in: http://extensionpublications.unl.edu/assets/html/ g1409/build/g1409.htm

26. Mader TL, Johnson LJ, Gaughan JB. 2010. A comprehensive index for assessing environmental stress in animals. J Anim Sci 88: 2153-2165. doi: 10.2527/jas.2009-2586

27. Manterola C, Otzen T. 2014. Estudios observacionales. Los diseños utilizados con mayor frecuencia en investigación clínica. Int J Morphol 32: 634-645.

28. Montelli NLLL, Macitelli F, da Silva Braga J, da Costa MJRP. 2019. Economic impacts of space allowance per animal on beef cattle feedlot. Semina: Ciências Agrárias 40(Supl 3): 3665-3678. doi: 10.5433/1679-0359.2019v40n6supl3p3665

29. Nienaber JA, Hahn GL. 2007. Livestock production system management responses to thermal challenges. Int J Biometeorol 52: 149-57. doi: 10.1007/s00484-007-0103-x/217

30. Olivares BO, Guevara E, Oliveros $Y$, López L. 2013. Aplicación del índice de confort térmico como estimador del estrés calórico en la producción pecuaria de la Mesa de Guanipa, Anzoátegui, Venezuela. Zootec Trop 31: 209-223.

31. Pelley M, Lirette A, Tennessen T. 1995. Observations on the responses of feedlot cattle to attempted environmental 
enrichment. Can J Anim Sci 75:631-632. doi: 10.4141/cjas95-093

32. Ratnakan AP, Sejian V, Sanjo Jose V, Vaswani S, Bagath M, Krishnan G, Beena V, et al. 2017. Behavioral response to livestock adaptation to heat stress challenges. A review. Asian J Anim Sci 11: 1-13. doi: 10.3923/ajas.2017.1.13

33. Renaudeau D, Collin A, Yahav S, Basilio V, Gourdine JL, Collier RJ. 2012. Adaptation to hot climate and strategies to alleviate heat stress in livestock production. Animals 6: 707-728. doi: 10.1017/S1751731111002448

34. Romo-Valdez A, Pérez-Linares C, Figueroa-Saavedra F, Portillo-Loera J, Ríos-Rincón F. 2019. Respuesta conductual de bovinos productores de carne en finalización intensiva en clima desértico cálido. Abanico Vet 9: 1-18. doi: 10.21299/abavet2019.28

35. Sullivan ML, Cawdell-Smith AJ, Made TL, Gaughan JB. 2011. Effect of shade area on performance and welfare of short-fed feedlot cattle. J Anim Sci 89: 2911-2925. doi: 10.2527/ jas.2010-3152

36. Tucker CB, Coetzee JF, Stookey JM, Thompson DV, Grandin T, Schwartzkopf-Genswein KF. 2015. Beef cattle welfare in USA: identification of priorities for future research. Anim Health Res Rev 16: 107-124. doi: 10.1017/S1466252315000171

37. Thornton PK. 2010. Livestock production: recent trends, future prospects. Philos T R Soc A 365: 28532867. doi: 10.1098/rstb.2010.0134 